\title{
Stereology of Microstructural Dihedral Angles in Three-Dimensions
}

\author{
A. M. Gokhale and S. Zhang
}

School of Materials Science and Engineering, Georgia Institute of Technology, Atlanta, GA 30332

Three-dimensional (3D) material microstructures often contain grain boundaries and/or interfaces, which are two-dimensional (2D) features. Junctions of such 2D features create one-dimensional edges (for example, grain edges, which are junctions of three grain boundaries). When grain boundaries (and/or interfaces) meet at an edge they also create microstructural angle(s) called dihedral angle(s). The local dihedral angle formed by two microstructural interfaces/surfaces where they meet to form an edge is defined as the dihedral angle formed by their tangent planes at the edge element. The linear angle formed by intersection of the dihedral angle with the plane perpendicular to the edge element is a quantitative measure of that dihedral angle. In the 3D microstructures containing lineal edges such as grain edges in polycrystalline materials and necks in liquid-phase sintered microstructures, a distribution of dihedral angles usually exists. Dihedral angle distribution and corresponding mean dihedral angle are of fundamental importance in the science of microstructures. In the materials processes such as liquidphase sintering, welding, and brazing, where a liquid phase is present, the microstructural evolution significantly depends on the wetting of the solid surfaces by the liquid phase, which is primarily controlled by the dihedral angles. In liquid-phase sintering, smaller dihedral angles or wetting angles facilitate spreading of the liquid over the surfaces of the solid grains and promote densification but increase distortion in the liquid-phase sintered alloys [1]. Dihedral angle distribution also affects contiguity and topological connectivity of liquid-phase sintered microstructures [2]. Further, under thermodynamic equilibrium conditions, the dihedral angles are related to the corresponding interfacial energies. Therefore, stereological estimation of 3D dihedral angle distributions is of interest.

Direct assumption-free measurements of true 3D dihedral angles are difficult. An apparent section dihedral angle observed in a 2D metallographic plane depends on the corresponding true 3D dihedral angle and the angular orientation of the sectioning plane, and therefore, it is not necessarily equal to the corresponding true 3D dihedral angle. As a result, a distribution of apparent 2D section dihedral angles is usually observed in random 2D metallographic planes even when there is only one true 3D dihedral angle. Consequently, stereological transforms are required to estimate the attributes of a 3D dihedral angle distribution from the measurements of apparent section angles performed in 2D metallographic planes. It can be shown that even when a distribution of 3D dihedral angles exists, the mean value of the population of apparent section angles $\langle\Psi\rangle$ observed in isotropic uniform random (IUR) 2D planes is equal to the mean value $\langle\chi\rangle$ of the distribution of 3D dihedral angles in the microstructure [3].

$$
<\chi><\Psi>
$$

Therefore, the mean value of 3D dihedral angles can be estimated from the measurements of the apparent section angles $\Psi$ in the IUR 2D sections. Nonetheless, the mean value and at least a measure of the spread (such as variance) are needed to characterize a distribution of 3D dihedral angles. Therefore, estimation of at least one more independent attribute of 3D dihedral angle distribution is desirable. This contribution presents a new general stereological relationship for estimation of the mean value of the sine of the dihedral angles in a $3 \mathrm{D}$ dihedral angle distribution, $<\sin \chi>$ from the measurements of apparent section angles $\psi$ in the IUR 2D sections, which is given as follows. 


$$
<\sin \chi>=(1 / \pi)<\left[\Psi(\pi-\Psi)+\sin ^{2} \Psi\right]>
$$

In equation (2), $<\left[\Psi(\pi-\Psi)+\sin ^{2} \Psi\right]>$ is the mean value of the quantity $\left[\Psi(\pi-\Psi)+\sin ^{2} \Psi\right]$ in the IUR 2D sections, which can be estimated from the experimental measurements of the apparent section angles $\Psi$. Consequently, if a two-parameter distribution function (for example, normal distribution) can be assumed to represent the 3D dihedral angle distribution, the mean and the variance (a measure of spread) of such 3D dihedral angle distribution can be calculated by using equations (1) and (2). Thus, equations (1) and (2) can be utilized to characterize evolution of 3D dihedral distributions during materials processes such grain growth and liquid-phase sintering. In the present work, these stereological transforms are applied to the experimental data on the distribution of apparent section angles of abutting $\mathrm{W}$ grains in a liquid-phase sintered microstructure of a W-Ni-Fe alloy [4]. The microstructure of the alloy liquid-phase sintered for 45 minutes at $1500 \mathrm{C}$ is shown in Figure 1(a). The experimentally measured distribution of apparent $2 \mathrm{D}$ section angles at the necks between the abutting $\mathrm{W}$ grains is shown in Figure 1(b). The 2D distribution was obtained from the measurements of 9,700 section angles using automated digital image processing [4]. The mean and the variance of the 3D dihedral distribution can be calculated from such information if it can be assumed that the 3D dihedral distribution can be represented by a two parameter distribution like normal distribution.

\section{References:}

[1] R.M. German, J. Mater. Sci. 44 (2009), p. 1.

[2] J.L. Johnson, A. Upadhyaya, and R.M. German, Metall. \& Mater. Trans. B. 29B (1998), p. 857.

[3] R.T. DeHoff, Metallography, 19 (1986), p. 209.

[4] W.B. Goodwin, M. Bharadwaj, Y. Mao, et al, Scripta Materialia, 61 (2009), p. 1101.

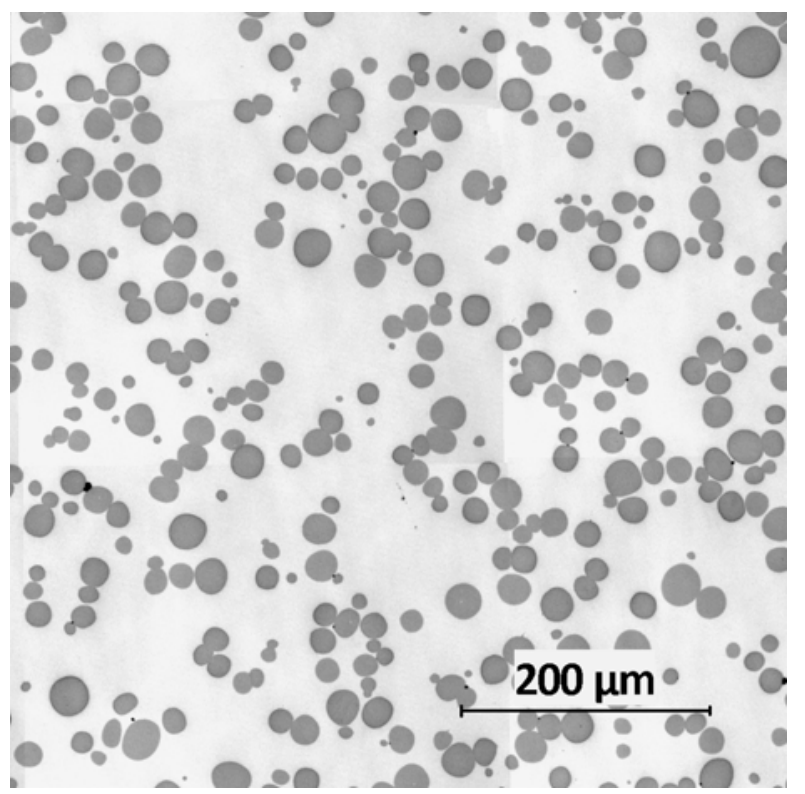

(a)

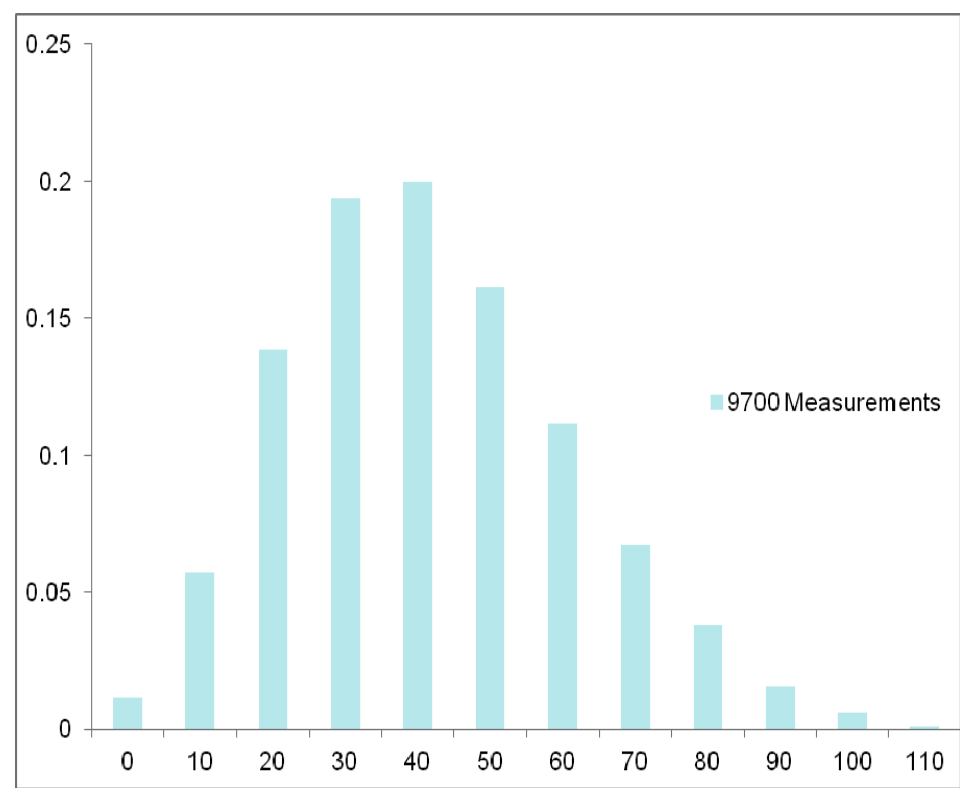

(b)

Figure 1: (a) Microstructure of a W-Ni-Fe alloy liquid-phase sintered at $1500 \mathrm{C}$ for 1 minute. (b) Experimentally measured distribution of apparent section dihedral angles in 2D section obtained from the measurements of 9700 section angles using automated digital image processing. 